\title{
Continuous Use of Thienopyridine May Be as Safe as Low-Dose Aspirin in Endoscopic Resection of Gastric Tumors
}

\author{
Sooyeon $\mathrm{Oh}^{1}$, Sang Gyun $\mathrm{Kim}^{1}$, Jung Kim${ }^{1}$, Ji Min $\mathrm{Choi}^{2}$, Joo Hyun Lim²${ }^{2}$, Hyo-Joon Yang ${ }^{3}$, Jae Yong Park ${ }^{4}$, Seung Jun Han ${ }^{1}$, \\ Jue Lie Kim ${ }^{1}$, Hyunsoo Chung $^{1}$, and Hyun Chae Jung ${ }^{1}$ \\ ${ }^{\prime}$ Department of Internal Medicine and Liver Research Institute, Seoul National University College of Medicine, ${ }^{2}$ Department of Internal \\ Medicine, Healthcare Research Institute, Seoul National University Hospital Healthcare System Gangnam Center, ${ }^{3}$ Division of Gastroenterology, \\ Department of Internal Medicine and Gastrointestinal Cancer Center, Kangbuk Samsung Hospital, Sungkyunkwan University School of \\ Medicine, and ${ }^{4}$ Department of Internal Medicine, Chung-Ang University Hospital, Chung-Ang University College of Medicine, Seoul, Korea
}

See editorial on page 369.

Background/Aims: Current guidelines recommend withholding antiplatelets for 5-7 days before high-risk endoscopic procedures. We investigated whether this reduces post-endoscopic submucosal dissection (ESD) bleeding. Methods: Gastric ESD cases with antiplatelets were retorospectively reviewed. Withholding antiplatelets for 5-7 days before ESD was defined as cessation and 0-4 days as continuation. The rate and risk of post-ESD bleeding according to the types and cessation of antiplatelets were assessed. Results: Among the 215 patients (117 adenoma and 98 early gastric cancer), 161 patients were on single (94 aspirin, 56 thienopyridine, and 11 other agents), 51 on dual, and 3 on triple antiplatelets. Post-ESD bleeding rates were $12.8 \%$ in aspirin users, $3.6 \%$ in thienopyridine, $27.5 \%$ in dual, 33.3\% in triple therapy, and $9.7 \%$ in the cessation and $15.0 \%$ in the continuation group. Multiple antiplatelets (odds ratio [OR], 2.41; 95\% confidence interval [Cl], 1.01 to 5.76) and specimen size $\geq 5.5 \mathrm{~cm}$ (OR, 2.84; 95\% Cl, 1.04 to 7.73) were the risk of bleeding, while continuation of thienopyridine (OR, 0.23; 95\% $\mathrm{Cl}, 0.05$ to 1.09) and antiplatelets (OR, 1.83; 95\% Cl, 0.68 to 4.94) did not increase the risk of bleeding. Conclusions: Continuing thienopyridine and aspirin did not increase the risk of post-ESD. Multiple antiplatelet therapy and a large specimen size were independent risk factors of post-ESD bleeding. (Gut Liver 2018;12:393-401)

Key Words: Antiplatelet; Aspirin; Thienopyridine; Endoscopic submucosal dissection; Post-endoscopic submucosal dissection bleeding

\section{INTRODUCTION}

The use of antiplatelet agents, perioperatively, during highrisk endoscopic procedures is concerning. One of the debated high-risk endoscopic procedures is endoscopic submucosal dissection (ESD) of gastric tumors. ESD is a very efficient curative modality for the treatment of early gastric cancer (EGC) and gastric dysplasia. However, post-ESD bleeding is a major adverse event, and the rate of bleeding ranges between $0 \%$ and $15.6 \% .^{1-5}$ Antiplatelet therapy affects the occurrence of postESD bleeding. ${ }^{4,5}$

In patients on antiplatelet therapy, the risk of thrombosis during cessation of the antiplatelet agents should be carefully considered, and guidelines proposed by the American Society for Gastrointestinal Endoscopy (ASGE) and the European Society for Gastrointestinal Endoscopy have been changing accordingly. In the past, discontinuing antiplatelet agents 7 to 10 days before high-risk endoscopic procedures was recommended. ${ }^{6}$ However, cessation of antiplatelet agents increases the risk of fatal thrombosis, such as cerebral infarction and coronary artery stent thrombosis. ${ }^{3,7}$ The guidelines responded to these fatal outcomes by gradually reducing the cessation period to 5-7 days and, in turn, recommending the continuation of low-dose aspirin monotherapy. ${ }^{8-12}$ However, adverse thrombotic events were reported even when periprocedural management complied with the most recent guidelines. ${ }^{13,14}$

As of now, the guidelines are not finalized and are yet to

Correspondence to: Sang Gyun Kim

Division of Gastroenterology, Department of Internal Medicine and Liver Research Institute, Seoul National University College of Medicine, 101 Daehak-ro, Jongno-gu, Seoul 03080, Korea

Tel: +82-2-740-8112, Fax: +82-2-743-6701, E-mail: harley1333@hanmail.net

Received on August 23, 2017. Revised on October 14, 2017. Accepted on October 25, 2017. Published online February 13, 2018 pISSN 1976-2283 eISSN 2005-1212 https://doi.org/10.5009/gnl17384

@ This is an Open Access article distributed under the terms of the Creative Commons Attribution Non-Commercial License (http://creativecommons.org/licenses/by-nc/4.0) which permits unrestricted non-commercial use, distribution, and reproduction in any medium, provided the original work is properly cited. 
be optimized. Formulating recommendations is challenging because of the scarcity of high-quality evidence. ${ }^{8}$ One clinical trial where the study protocol conformed to a guideline proved the difficulty in performing a high-quality study of this topic. The study evaluated the effect of thienopyridine derivatives on post-ESD bleeding. Although the study withdrew thienopyridine derivatives 5 to 7 days before the procedure, as recommended in the Japan Gastroenterological Endoscopy Society guideline, ${ }^{10}$ it was terminated at an early phase because the rate of adverse major bleeding events exceeded the predetermined safety criteria $(25 \%, 7 / 28) .{ }^{15}$ The results of a recent clinical trial have prompted clinicians to question whether performing gastric ESD is possible without discontinuation of the single antiplatelet of thienopyridine derivatives. ${ }^{16}$ In that study, only 10 patients were enrolled and post-ESD bleeding occurred in two patients. As such, data from clinical trials are scarce, and most of the evidence comes from retrospective observational studies composed of a small number of study subjects.

There are several unanswered questions. One is whether thienopyridine poses a higher bleeding risk compared to low-dose aspirin, since evidence collected so far suggested otherwise. ${ }^{13}$ Another is whether the current recommendation of a withdrawal period of 5 to 7 days according to current guidelines does indeed reduce the bleeding risk and is simultaneously safe enough to prevent thrombosis. Moreover, optimal periprocedural management for those who are on multiple antiplatelet agents and cannot defer elective endoscopic procedures is yet to be determined. This is especially important since the patients on multiple antiplatelet agents are at a very high-risk of thrombosis.

Hence, the aim of this study was to investigate the post-ESD bleeding rate according to the different types of antiplatelet therapy and according to the duration of antiplatelet cessation before ESD in patients taking antiplatelet agents with definite indications. To estimate the bleeding risk more accurately, we aimed to adjust these antiplatelet factors with other factors, which are known to contribute to the risk of bleeding.

\section{MATERIALS AND METHODS}

\section{Patients}

Patients who underwent ESD for gastric tumors at Seoul National University Hospital (SNUH, Seoul, Korea) between April 2005 and November 2016 were retrospectively reviewed. The patients who were on antiplatelet therapy but not on anticoagulation were screened. The indication for antiplatelet therapy was meticulously reviewed and patients who did not have a definite indication, namely those on antiplatelet agents for primary prevention, were excluded. Periprocedural management of antiplatelet agents was discussed with the prescribing specialists and their recommendations were followed. Patients who had discontinued antiplatelet agents more than 7 days before ESD were excluded. Most patients resumed antiplatelet agents 1 to 3 days after ESD. Some patients discontinued antiplatelet agents for a longer period, and those patients who did so for reasons other than post-ESD bleeding were excluded as well. For all patients, a double-dose of a proton pump inhibitor was administered intravenously on the day of ESD and orally from the next day for 4 weeks. Regarding diet, patients were maintained non per os on the day of ESD. The next morning, patients were allowed to take sips of water. If the patient exhibited no adverse response to drinking water, they were allowed to proceed with a semifluid diet followed by a soft blended diet (SBD). If there were no signs of adverse events, patients were discharged a day after ESD. Upon discharge, patients were taught to consume an SBD for at least one week before proceeding to a normal regular diet. The clinical course of patients was reviewed up to at least 6 months after ESD under the assumption that any event happening during that period may have relevance to the ESD procedure. This study was approved by the Institutional Review Board of SNUH (IRB number H-1612-081-814), and was conducted in accordance with the Declaration of Helsinki. Patient consent was waived, because of the retrospective nature of this study.

\section{ESD procedure}

ESD was performed using an IT-knife (Helmet Snare; Kachu Technology Co. Ltd., Seoul, Korea) through a standard singlechannel endoscope (Olympus H260; Olympus Optical, Tokyo, Japan), as previously reported. ${ }^{17}$ After chromoendoscopic observation with indigo carmine, the tumor was marked by dotting $5 \mathrm{~mm}$ outside the tumor margin with argon plasma coagulation (VIO 300D; Erbe Elektromedizin GmbH, Tübingen, Germany). Then, a mixture of normal saline solution and indigo carmine with diluted epinephrine $(1: 100,000)$ was injected into the submucosal layer. After making a small initial incision with a needle-knife, a circumferential mucosal incision was made around the marking dots, and the submucosal layer was dissected using the IT-knife in 80-W endocut mode. During the ESD, hemostasis was performed for bleeding spots or visible vessels with a Coagrasper (MTN BF-2; Standard Sci-Tech Inc., Seoul, Korea).

The endoscopically resected specimen was extended on a polystyrene board with pins and fixed in 10\% formalin immediately. It was sectioned serially at 2-mm intervals, stained with hematoxylin-eosin, and subjected to histological evaluation. Histological type of the tumor, size, depth of invasion, and capillary involvement were evaluated according to the Japanese Classification of Gastric Carcinoma. ${ }^{18}$ Complete resection was defined as tumor-free areas in both the horizontal and the vertical margins. All the cases included in this study were resected en-bloc.

\section{Data analysis}

Some patients had received multiple ESDs due to synchronous or metachronous lesions. For those patients, only one 
lesion per patient was included for analyses. In particular, the lesion was selected if it had a post-ESD bleeding event or was larger.

In this study, withholding antiplatelet agents 5 to 7 days before ESD was defined as cessation or discontinuation. Withholding 0 to 4 days was defined as continuation. In case of multiple antiplatelet therapy, withholding all agents 0 to 4 days was defined as continuation, and withholding any agent 5 days or longer was defined as cessation.

Post-ESD bleeding was defined as any episode of the following: overt hematemesis/hematochezia, a drop in hemoglobin of $2 \mathrm{~g} / \mathrm{dL}$, or needing endoscopic hemostasis or angiographic embolization and/or transfusion. ${ }^{3}$ Early bleeding was defined as a bleeding episode within 72 hours after ESD, and late bleeding was defined as such beyond 72 hours. ${ }^{3}$ Some patients experienced re-bleeding events, but only the first episodes of bleeding were included for analyses.

Information on factors, which may influence post-ESD bleeding, was collected. Especially, comorbidity, which may affect coagulative function, was carefully screened. Such comorbidities were predefined as follows: liver cirrhosis (Child-Pugh class B or C), chronic kidney disease (CKD) (CKD stage IV or V), and he- matologic disease accompanying an abnormal level of platelets. Coagulation abnormality was defined as abnormal laboratory findings of activated partial thromboplastin time or prothrombin time and a platelet count of $<100,000 / \mathrm{mm}^{3}$.

\section{Statistical analysis}

Continuous variables were analyzed with Student t-test and were transformed into categorical variables with cutoff values that were indicated as being optimal according to receiver operating characteristic (ROC) curve analysis and Youden's index ( $\mathrm{J}=$ sensitivity+specificity-1). Categorical variables were compared using the Pearson chi-square test or Fisher exact test, as appropriate. Variables that showed statistical significance or those that were considered clinically important were subjected to univariate and multivariate logistic regression analysis, with appropriate categorization. IBM SPSS statistics version 21.0 (IBM Corp., Armonk, NY, USA) was used for all statistical analyses.

\section{RESULTS}

\section{Clinicopathological characteristics of the participants}

In total, 5,305 ESD cases performed at SNUH between April

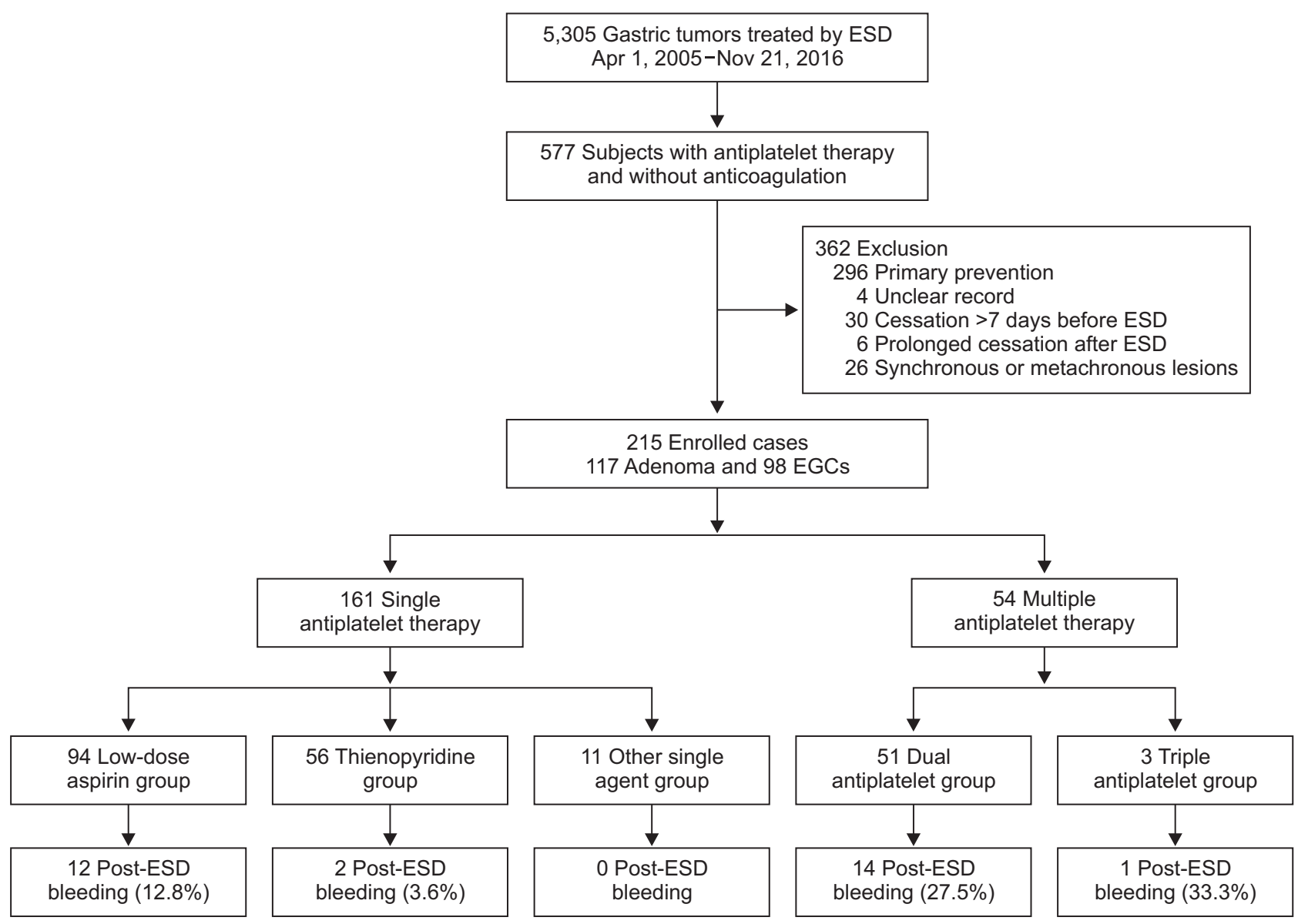

Fig. 1. Patient flowchart.

EDS, endoscopic submucosal dissection; EGCs, early gastric cancers. 
2005 and November 2016 were reviewed. We screened 577 cases on antiplatelet therapy and without anticoagulation. We excluded 296 cases that were on antiplatelet therapy for primary prevention, four cases because of unclear records of their cessation periods, 30 cases because of cessation periods exceeding 7 days before ESD, six cases that discontinued antiplatelet agents more than 3 days after ESD for reasons other than postESD bleeding, 13 cases of synchronous lesions, and 13 cases of metachronous lesions. Overall, 215 cases of gastric neoplasms (117 adenoma and 98 EGC) were included (Fig. 1). Only one

Table 1. Baseline Characteristics of Gastric Endoscopic Submucosal Dissection Cases

\begin{tabular}{|c|c|}
\hline Variable & Value \\
\hline Male sex & $173(80.5)$ \\
\hline Age, yr & 70 (49-85) \\
\hline \multicolumn{2}{|l|}{ Types of antiplatelet therapy } \\
\hline Low-dose aspirin & $94(43.7)$ \\
\hline Thienopyridine & $56(26.0)$ \\
\hline Other single agent & $11(5.1)$ \\
\hline Dual agents & $51(23.7)$ \\
\hline Triple agents & $3(1.4)$ \\
\hline \multicolumn{2}{|l|}{ Cessation period, day } \\
\hline Continuation (0-4) & $153(71.2)$ \\
\hline Cessation (5-7) & $62(28.8)$ \\
\hline \multicolumn{2}{|c|}{ Indications for antiplatelet therapy } \\
\hline Coronary artery disease & $99(46.0)$ \\
\hline Cerebrovascular disease & $76(35.3)$ \\
\hline Atrial fibrillation & $7(3.3)$ \\
\hline Other vascular disease & $15(7.0)$ \\
\hline Multiple of the above & $18(8.4)$ \\
\hline Size of specimen, cm & $4.3(2.5-10.6)$ \\
\hline \multicolumn{2}{|l|}{ Final pathology } \\
\hline No tumor & $1(0.5)$ \\
\hline Adenoma & $117(54.4)$ \\
\hline Differentiated cancer & $93(43.3)$ \\
\hline Undifferentiated cancer & $4(1.9)$ \\
\hline \multicolumn{2}{|l|}{ Resection margin } \\
\hline Complete resection & 199 (92.6) \\
\hline Incomplete resection & $16(7.4)$ \\
\hline \multicolumn{2}{|l|}{ Location } \\
\hline Upper & $23(10.7)$ \\
\hline Middle & $56(26.0)$ \\
\hline Lower & $136(63.3)$ \\
\hline \multicolumn{2}{|l|}{ Tumor type } \\
\hline Elevated & 78 (36.3) \\
\hline Flat & $87(40.5)$ \\
\hline Depressed & $50(23.3)$ \\
\hline
\end{tabular}

Data are presented as number (\%) or median (range). patient of the included cases had microperforation. The perforation was managed conservatively without surgical intervention.

Population characteristics are summarized in Table 1. Among 215 patients, 161 were on single (94 low-dose aspirin, 56 thienopyridine, five cilostazol, five triflusal, and one sarpogrelate),

Table 2. Post-ESD Bleeding Rates and Univariate Risk Analyses

\begin{tabular}{|c|c|c|}
\hline Variable & $\begin{array}{l}\text { No. of post-ESD } \\
\text { bleeding events (\%) }\end{array}$ & $\mathrm{p}$-value \\
\hline Sex & & 0.738 \\
\hline Female & $5(11.9)$ & \\
\hline Male & 24 (13.9) & \\
\hline Age, yr & & 0.551 \\
\hline$<79$ & $24(12.8)$ & \\
\hline$\geq 79$ & $5(17.9)$ & \\
\hline Types of antiplatelet therapy & & 0.003 \\
\hline Low-dose aspirin & $12(12.8)$ & \\
\hline Thienopyridine & $2(3.6)$ & \\
\hline Other single agent & 0 & \\
\hline Dual agents & $14(27.5)$ & \\
\hline Triple agents & $1(33.3)$ & \\
\hline Cessation period, day & & 0.298 \\
\hline Continuation (0-4) & $23(15.0)$ & \\
\hline Cessation (5-7) & $6(9.7)$ & \\
\hline Indications for antiplatelet therapy & & 0.455 \\
\hline Coronary artery disease & $16(16.2)$ & \\
\hline Cerebrovascular disease & $10(13.2)$ & \\
\hline Atrial fibrillation & 0 & \\
\hline Other vascular disease & 0 & \\
\hline Multiple of the above & $3(16.7)$ & \\
\hline Size of specimen, $\mathrm{cm}$ & & 0.035 \\
\hline$<5.5$ & $21(11.3)$ & \\
\hline$\geq 5.5$ & 8 (27.6) & \\
\hline Final pathology & & 0.475 \\
\hline Adenoma & $14(12.0)$ & \\
\hline EGC & $15(15.3)$ & \\
\hline Resection margin & & 0.242 \\
\hline Complete resection & 25 (12.6) & \\
\hline Incomplete resection & $4(25.0)$ & \\
\hline Location & & 0.761 \\
\hline Upper & $3(13.0)$ & \\
\hline Middle & $6(10.7)$ & \\
\hline Lower & 20 (14.7) & \\
\hline Tumor type & & 0.652 \\
\hline Elevated & $9(11.5)$ & \\
\hline Flat & $14(16.1)$ & \\
\hline Depressed & $6(12.0)$ & \\
\hline
\end{tabular}

ESD, endoscopic submucosal dissection; EGC, early gastric cancer. 
51 on dual, and three on triple antiplatelet therapy. In combination therapies, aspirin or thienopyridine, or both were combined with one or two of the other antiplatelet agents mentioned above. The indications of antiplatelet therapy were coronary artery disease, cerebrovascular disease, atrial fibrillation, and other vascular problems. Among the included cases, none had a coagulation abnormality or predefined comorbidities that may influence coagulative function.

All tumors were resected en-bloc with grossly sufficient margins. In pathologic reports of the resected specimens, there were one no tumor, 117 adenomas, 93 differentiated adenocarcinomas, and four undifferentiated adenocarcinomas. The no tumor case had been initially diagnosed as well-differentiated adenocarcinoma by biopsy. Therefore, it was grouped into the EGC group together with differentiated and undifferentiated adenocarcinomas for further analyses.

\section{Post-ESD bleeding rates}

Among the 215 cases, post ESD-bleeding episodes occurred in 29 (13.5\%). Post-ESD bleeding rates according to each factor are

Table 3 . Post-Endoscopic Submucosal Dissection Bleeding Rates According to the Exact Cessation Period in Single Antiplatelet Therapy

\begin{tabular}{cccc}
\hline $\begin{array}{c}\text { Cessation } \\
\text { period, day }\end{array}$ & $\begin{array}{c}\text { Low-dose } \\
\text { aspirin }\end{array}$ & Thienopyridine & $\begin{array}{c}\text { Other } \\
\text { single agent }\end{array}$ \\
\hline 0 & $4 / 26(15.4)$ & $2 / 28(7.1)$ & $0 / 6(0)$ \\
1 & $1 / 8(12.5)$ & $0 / 5(0)$ & $0 / 1(0)$ \\
2 & $1 / 6(16.7)$ & $0 / 3(0)$ & $0 / 2(0)$ \\
3 & $1 / 10(10.0)$ & $0 / 4(0)$ & $0 / 2(0)$ \\
4 & $1 / 7(14.3)$ & $0 / 5(0)$ & - \\
5 & $1 / 17(5.9)$ & $0 / 4(0)$ & - \\
6 & $2 / 5(40.0)$ & $0 / 2(0)$ & - \\
7 & $1 / 15(6.7)$ & $0 / 5(0)$ & - \\
\hline
\end{tabular}

Data are presented as number/total number (\%). presented with the results of univariate risk analyses in Table 2. Age and specimen size variables were tested as categorical variables with cutoff values to define higher and lower risk groups for post-ESD bleeding.

In low-dose aspirin users, the bleeding rate was 12.8\% (12/94), which was higher than that of thienopyridine users $(3.6 \%, 2 / 56)$. The number of patients on a single antiplatelet therapy with cilostazol, triflusal, or sarpogrelate was very limited $(n=11)$, and post-ESD bleeding episodes were not observed in these patients.

Table 4. Post-ESD Bleeding Rates According to the Exact Cessation Period in Multiple Antiplatelet Therapy

\begin{tabular}{cc}
\hline Cessation period, day & Post-ESD bleeding events \\
\hline X0Y0 & $4 / 16(25.0)$ \\
X1Y0 & $2 / 2(100)$ \\
X1Y1 & $1 / 6(16.7)$ \\
X2Y1 & $0 / 1(0)$ \\
X2Y2 & $0 / 1(0)$ \\
X3Y0 & $0 / 1(0)$ \\
X3Y3 & $2 / 2(100)$ \\
X4Y0 & $0 / 1(0)$ \\
X4Y4 & $2 / 4(50.0)$ \\
X5Y0 & $0 / 1(0)$ \\
X5Y5 & $0 / 4(0)$ \\
X6Y0 & $1 / 2(50.0)$ \\
X6Y6 & $1 / 4(25.0)$ \\
X7Y7 & $1 / 6(16.7)$ \\
X0Y0Z0 & $1 / 3(33.3)$ \\
\hline
\end{tabular}

Data are presented as number/total number (\%).

$\mathrm{X}$ indicates the antiplatelet agent that was discontinued first. $\mathrm{Y}$, and $\mathrm{Z}$ indicate the antiplatelet agents that were discontinued subsequently or continued. The number followed by characters $\mathrm{X}, \mathrm{Y}$, and $\mathrm{Z}$ indicates the cessation period in days.

ESD, endoscopic submucosal dissection.

Table 5. Risk Factors of Post-Endoscopic Submucosal Dissection Bleeding in Univariate and Multivariate Logistic Regression Analyses

\begin{tabular}{|c|c|c|c|c|}
\hline & \multicolumn{2}{|c|}{ Univariate logistic regression } & \multicolumn{2}{|c|}{ Multivariate logistic regression } \\
\hline & OR (95\% CI) & p-value & OR $(95 \%$ CI) & $\mathrm{p}$-value \\
\hline \multicolumn{5}{|c|}{ Type of antiplatelet therapy } \\
\hline Low-dose aspirin & 1 & & 1 & \\
\hline Thienopyridine & $0.25(0.05-1.18)$ & 0.080 & $0.23(0.05-1.09)$ & 0.065 \\
\hline Dual/triple agents & $2.63(1.12-6.15)$ & 0.026 & $2.41(1.01-5.76)$ & 0.049 \\
\hline \multicolumn{5}{|l|}{ Cessation period, day } \\
\hline Cessation (5-7) & 1 & & 1 & \\
\hline Continuation (0-4) & $1.65(0.64-4.28)$ & 0.302 & $1.83(0.68-4.94)$ & 0.231 \\
\hline \multicolumn{5}{|l|}{ Size of specimen, $\mathrm{cm}$} \\
\hline$<5.5$ & 1 & & 1 & \\
\hline$\geq 5.5$ & $2.99(1.18-7.60)$ & 0.021 & $2.84(1.04-7.73)$ & 0.041 \\
\hline
\end{tabular}

OR, odds ratio; CI, confidence interval. 


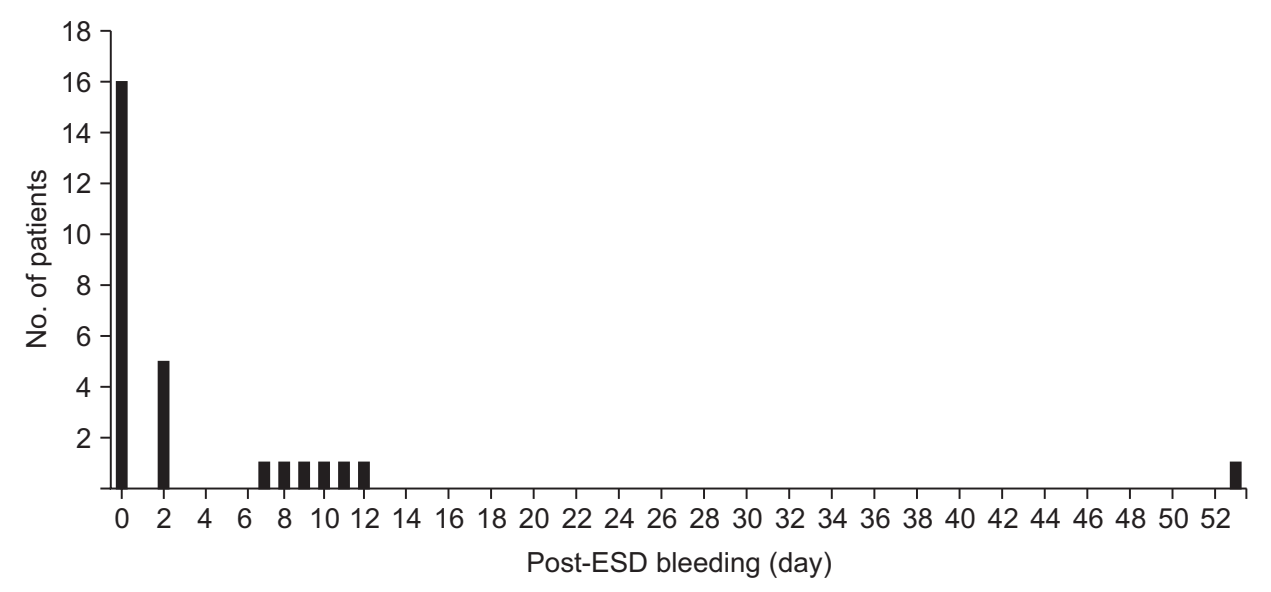

Fig. 2. Time points of post-endoscopic submucosal dissection (ESD) bleeding episodes depicted as a histogram.
In combination antiplatelet therapies, the post-ESD bleeding rates were much higher. The bleeding rate was 27.5\% (14/51) in dual therapy and 33.3\% (1/3) in triple therapy. In terms of the cessation period, the post-ESD bleeding rate was higher in the continuation group $(15.0 \%, 23 / 153)$ than in the cessation group (9.7\%, 6/62), but the difference was not statistically significant $(\mathrm{p}=0.298)$. Detailed data on post-ESD bleeding rates according to exact cessation period and types of antiplatelet therapy are presented in Tables 3 and 4.

Among other factors, the bleeding rate was significantly higher in patients with a specimen size $\geq 5.5 \mathrm{~cm}(27.6 \%, 8 / 29)$ than in patients with a specimen size $<5.5 \mathrm{~cm}(11.3 \%, 21 / 186$, $\mathrm{p}=0.035$ ). The other factors failed to show statistical significance.

\section{Risk factors for post-ESD bleeding}

Variables with clinical significance were tested with univariate and multivariate logistic regression analyses (Table 5). In multivariate logistic regression analyses, multiple antiplatelet therapy was significantly associated with a higher risk of postESD bleeding (odds ratio [OR], 2.41; 95\% confidence interval [CI], 1.01 to 5.76), but thienopyridine monotherapy was not (OR, 0.23; 95\% CI, 0.05 to 1.09), compared to monotherapy with low-dose aspirin. Continuation of antiplatelet therapy did not result in a statistically significant difference in the risk of postESD bleeding (OR, 1.83; 95\% CI, 0.68 to 4.94) compared to cessation. Specimen size $\geq 5.5 \mathrm{~cm}$ was associated with an increased risk of bleeding (OR, 2.84; 95\% CI, 1.04 to 7.73).

\section{Management of post-ESD bleeding}

Among the patients who had episodes of post-ESD bleeding $(n=29)$, one patients visited and were managed at other hospitals. The rest visited and were managed at SNUH. Most of the post-ESD bleeding was early bleeding (72.4\%, 21/29). Time points of the bleeding events are depicted as a histogram in Fig. 2.

Among the 28 bleeding cases that were managed at SNUH, 22 patients underwent esophagogastroduodenoscopy (EGD).
Table 6. Management of the First Episodes of Post-Endoscopic Submucosal Dissection Bleeding Events

\begin{tabular}{lcl}
\hline \multicolumn{1}{c}{ Management methods } & $\begin{array}{c}\text { No. of } \\
\text { patients }\end{array}$ & $\begin{array}{c}\text { Hemoglobin } \\
\text { drop, g/dL }\end{array}$ \\
\hline Managed outside & 1 & \\
Conservative management & 5 & $2.70 \pm 1.46$ \\
Transfusion only & 1 & 2.7 \\
Endoscopy only & 6 & $2.55 \pm 2.12$ \\
Endoscopy+transfusion & 6 & $4.42 \pm 1.75$ \\
Endoscopy+hemostasis & 6 & $2.12 \pm 1.57$ \\
Endoscopy+transfusion+hemostasis & 4 & $3.43 \pm 1.07$ \\
\hline
\end{tabular}

Data are presented as mean \pm standard error.

During EGD, 10 patients had active bleeding lesions in the iatrogenic ulcer bases and received endoscopic hemostasis (eight patients with coagulation, one with argon plasma coagulation, and one with coagulation combined with hemoclipping), and 12 patients had no active bleeding focus requiring hemostasis. For example, one patient complaining of melena 53 days after ESD was found with an active iatrogenic ulcer crater covered with blood clots but without active bleeding in EGD. The other six patients were observed very closely, and they showed relatively stable vital signs and a tolerable drop in hemoglobin. Therefore, they could do without EGD and recovered well from their acute bleeding episodes. Detailed information on bleeding management methods and the levels of hemoglobin drop is presented in Table 6.

Re-bleeding occurred in four cases. Three patients were on dual antiplatelet therapy with low-dose aspirin and thienopyridine, while one patient was on triple antiplatelet therapy with low-dose aspirin, thienopyridine, and cilostazol. They were all in the continuation group. During EGD, three patients were found with active bleeding spots and received endoscopic hemostasis with coagulation, the other patient was without an active bleeding focus.

All of the initial bleeding and re-bleeding cases were man- 
aged effectively without having to undergo angiographic embolization or surgical hemostasis. There were no sequelae related to post-ESD bleeding.

\section{Thromboembolism}

Cerebral infarction occurred in one patient 4 months after ESD. The patient was an 84-year-old male. He was on thienopyridine monotherapy for angina pectoris. It was stopped 7 days before ESD and re-started 2 days after.

\section{DISCUSSION}

In this study, the rates of post-ESD bleeding according to different durations of antiplatelet cessation and the types of antiplatelet therapy were studied in the context of current guidelines. In addition, risk factors of post-ESD bleeding among antiplatelet users were investigated. The results of this study agree with those of previous studies. The overall rate of postESD bleeding in this study was $13.5 \%$ (29/215) which is approximately similar to those of other studies (11.6\% [20/172], ${ }^{3}$ 9.5\% [35/367], ${ }^{13}$ and 9.7\% [17/175] $]^{5}$. In multivariate analysis, thienopyridine monotherapy, compared to low-dose aspirin monotherapy, failed to show a significantly elevated risk of post-ESD bleeding. Interestingly, the trend was to the contrary. In terms of cessation period, antiplatelet continuation beyond the recommended cessation period ( 5 to 7 days before ESD) also failed to show statistical significance in bleeding risk. In the present study, only multiple antiplatelet therapy and specimen size $(\geq 5.5 \mathrm{~cm})$ were independent risk factors for post-ESD bleeding.

Thienopyridine is known to be more potent at inhibiting antiplatelet aggregation than low-dose aspirin, and it does not have the usual side effects of nonsteroidal anti-inflammatory drugs, such as drug allergy and gastrointestinal disturbance. Therefore, many cardiologist and neurologists often prefer thienopyridine over low-dose aspirin. The current guideline proposed by ASGE as well as the results of a recent meta-analysis both support the continuous use of low-dose aspirin in gastric ESD., ${ }^{8,19}$ If antiplatelet therapy should be continued, thienopyridine is recommended to be switched to low-dose aspirin monotherapy. Guidelines recommend low-dose aspirin over thienopyridine not because aspirin is proven to be safer, but because we have more experience with aspirin than thienopyridine. In the current study, the post-ESD bleeding rate with thienopyridine (3.6\%, 2/56) was much lower than that with low-dose aspirin $(12.8 \%, 12 / 94)$. When the risk was adjusted by cessation period and specimen size, thienopyridine did not exhibit a higher risk of bleeding than did low-dose aspirin. With low-dose aspirin as a reference group, the adjusted OR of thienopyridine toward the risk of post-ESD bleeding was 0.23 (95\% CI, 0.05 to 1.09; $\mathrm{p}=0.065$ ). There are similar findings in other studies. Igarashi et al. ${ }^{13}$ reported that the post-ESD bleeding rates with thienopyri- dine were $5.6 \%(1 / 18)$ in the antiplatelet continuation group and $12.5 \%(9 / 72)$ in the cessation group (cessation for 3 to 7 days before ESD), whereas those with low-dose aspirin were $12.1 \%(4 / 33)$ in the continuation group and 11.1\% (19/171) in the cessation group. Another study that evaluated the risks of upper gastrointestinal bleeding in a population based casecontrol study reported that the adjusted ORs for the risk of bleeding were 1.8 (95\% CI, 1.5 to 2.1) for low-dose aspirin use and 1.1 (95\% CI, 0.6 to 2.1) for clopidogrel use. ${ }^{20}$ The numbers of these results indicate that thienopyridine may carry a lower risk of bleeding compared to low-dose aspirin. Whether these observations are related to the inhibition of cyclooxygenase-1 by aspirin should be tested in future studies. For now, the results may well be interpreted that thienopyridine monotherapy failed to show a higher risk of bleeding than low-dose aspirin.

In previous studies, antiplatelet cessation for $<7$ days was shown to increase the risk of post-ESD bleeding. ${ }^{4,5}$ Therefore, antiplatelet cessation $\geq 7$ days is well-received to reduce the risk of bleeding. However, cessation for 5 to 7 days requires further research to understand the associated risks and benefits. Guidelines recommended 5 to 7 days since that is generally considered as the minimum duration of antiplatelet discontinuation that is required to allow restoration of normal platelet aggregability. ${ }^{21}$ However, there have been no studies indicating that 5 to 7 days actually reduces the bleeding risk compared to continuing antiplatelet therapy. In the current study, there were more bleeding events in the continuation group $(15.0 \%, 23 / 153)$ than in the cessation group $(9.7 \%, 6 / 62)$. However, the risk was not significantly elevated in multivariate logistic regression analysis (OR, 1.83; 95\% CI, 0.68 to 4.94). The observation was similar in the study by Igarashi et $a l,{ }^{13}$ although their cessation period differed ( 3 to 7 days). These observations may have been simply due to the small population size. However, there is a possibility that cessation of 5 to 7 days does not reduce the bleeding risk. It should be further evaluated in future studies.

The risk of post-ESD bleeding with multiple antiplatelet agents has been described in many studies. In the current study, the post-ESD bleeding rate with multiple agents was $27.8 \%$ (15/54) and the odds of post-ESD bleeding with multiple agents was 2.41 (95\% CI, 1.01 to 5.76). The bleeding rates in other studies were $35.5 \%(11 / 31)^{22}$ and $4.8 \%(1 / 21)$ in the continuation group and $16.2 \%(6 / 37)$ in the cessation group. ${ }^{13}$ It is known that dual antiplatelet therapy may confer a 3-fold increase in the risk of upper gastrointestinal bleeding. ${ }^{8,20,23,24} \mathrm{~A}$ recent meta-analysis showed that the discontinued multiple antithrombotic group had a significantly higher risk of bleeding than the discontinued single antithrombotic group (OR, 5.17; 95\% CI, 3.13 to 8.54). ${ }^{19}$ The guidelines recommend that all elective endoscopic procedures should be deferred until the patients can safely stop multiple antiplatelet therapy, particularly in patients with intracoronary stents. In practice, however, gastric ESD for EGC can hardly be deferred given the progressive na- 
ture and poor prognosis of gastric cancer. On the other hand, there are conditions that require life-long antiplatelet therapy and in which stopping antiplatelet therapy poses a fatal risk of thromboembolism. Examples of such cases included in this study were a patient who had suffered three episodes of stroke despite regular use of antiplatelet therapy and a patient who was recovering normally for 5 months after surviving from cardiac arrest. This latter patient had undergone coronary intervention twice with 1 month interval between the two interventions. For these patients, gastric ESDs were performed without stopping triple antiplatelet therapy since the consequences of thrombosis far outweighed those of bleeding.

In terms of size, specimen size $(>4 \mathrm{~cm})$ was associated with an increased risk of post-ESD bleeding in many studies. ${ }^{3-5,14,25,26}$ In the current analyses, a specimen size $\geq 5.5 \mathrm{~cm}$ significantly increased the bleeding risk (OR, 2.84; 95\% CI, 1.04 to 7.73). The cutoff value $(5.5 \mathrm{~cm})$ that was used to define a higher risk group was acquired by ROC curve analysis. However, the numerical value of $5.5 \mathrm{~cm}$ itself is less important than the observation that a larger specimen size increases the risk of post-ESD bleeding.

The post-ESD bleeding cases, including re-bleeding cases, were all managed fairly well with the use of proton pump inhibitor with or without endoscopic hemostasis or blood transfusion. One case of thrombosis was observed during the clinical course. While it may be clinically relevant to ESD, a direct causal relationship is not evident since the event occurred far after ESD and the thrombotic event (cerebral infarction) was unrelated to the underlying disease (angina pectoris). Nevertheless, it is important to acknowledge that antiplatelet agents play protective roles against all thrombotic events in the elderly, regardless of the target organ. In the report by Igarashi et al., ${ }^{13}$ thrombotic events were observed to have occurred even when antiplatelet therapy was stopped 3, 6, and 7 days before ESD, which are within the range of current recommendations. In contrast, fatal cases of post-ESD bleeding in a patient with heparin replacement ${ }^{27}$ and in a patient on hemodialysis ${ }^{28}$ have been reported, where thrombotic events were the result of massive delayed bleeding. We believe that balancing the risks between thrombotic events and post-ESD bleeding in high-risk patients is important. As many previous studies indicate, ${ }^{13,16,29,30}$ continuing antiplatelet agents may be feasible, particularly when the patient is not using concomitant anticoagulation therapy nor exhibits any comorbidity that affects coagulative function.

Despite our careful analyses, there are some limitations to this study, including its retrospective design and small population size. We cannot deny the selection bias generated when we picked one lesion per patient in cases of synchronous and metachronous lesions. Despite these limitations, our research provides important insights into the risk of post-ESD bleeding according to antiplatelet use.

In conclusion, continuing thienopyridine monotherapy may be as safe as low-dose aspirin monotherapy during the peripro- cedural period of gastric ESD. Continuing antiplatelet therapy beyond the recommended period may not increase the risk of post-ESD bleeding significantly, and may be feasible to reduce the risk of thrombotic events. Multiple antiplatelet therapy and a larger specimen size are independent risk factors for post-ESD bleeding.

\section{CONFLICTS OF INTEREST}

No potential conflict of interest relevant to this article was reported.

\section{ACKNOWLEDGEMENTS}

This work was supported by a Grant from the Promising-Pioneering Researcher Program through Seoul National University in 2015.

\section{REFERENCES}

1. Oda I, Suzuki H, Nonaka S, Yoshinaga S. Complications of gastric endoscopic submucosal dissection. Dig Endosc 2013;25 Suppl 1:71-78.

2. Toyonaga T, Man-i M, East JE, et al. 1,635 Endoscopic submucosal dissection cases in the esophagus, stomach, and colorectum: complication rates and long-term outcomes. Surg Endosc 2013;27:1000-1008.

3. Lim JH, Kim SG, Kim JW, et al. Do antiplatelets increase the risk of bleeding after endoscopic submucosal dissection of gastric neoplasms? Gastrointest Endosc 2012;75:719-727.

4. Cho SJ, Choi IJ, Kim CG, et al. Aspirin use and bleeding risk after endoscopic submucosal dissection in patients with gastric neoplasms. Endoscopy 2012;44:114-121.

5. Koh R, Hirasawa K, Yahara S, et al. Antithrombotic drugs are risk factors for delayed postoperative bleeding after endoscopic submucosal dissection for gastric neoplasms. Gastrointest Endosc 2013;78:476-483.

6. Zuckerman MJ, Hirota WK, Adler DG, et al. ASGE guideline: the management of low-molecular-weight heparin and nonaspirin antiplatelet agents for endoscopic procedures. Gastrointest Endosc 2005;61:189-194.

7. Eisenberg MJ, Richard PR, Libersan D, Filion KB. Safety of shortterm discontinuation of antiplatelet therapy in patients with drugeluting stents. Circulation 2009;119:1634-1642.

8. ASGE Standards of Practice Committee, Acosta RD, Abraham NS, et al. The management of antithrombotic agents for patients undergoing GI endoscopy. Gastrointest Endosc 2016;83:3-16.

9. Veitch AM, Vanbiervliet G, Gershlick AH, et al. Endoscopy in patients on antiplatelet or anticoagulant therapy, including direct oral anticoagulants: British Society of Gastroenterology (BSG) and European Society of Gastrointestinal Endoscopy (ESGE) guidelines. Endoscopy 2016;48:385-402. 
10. Fujimoto K, Fujishiro M, Kato M, et al. Guidelines for gastroenterological endoscopy in patients undergoing antithrombotic treatment. Dig Endosc 2014;26:1-14.

11. ASGE Standards of Practice Committee, Anderson MA, BenMenachem T, et al. Management of antithrombotic agents for endoscopic procedures. Gastrointest Endosc 2009;70:1060-1070.

12. Boustière C, Veitch A, Vanbiervliet G, et al. Endoscopy and antiplatelet agents: European Society of Gastrointestinal Endoscopy (ESGE) guideline. Endoscopy 2011;43:445-461.

13. Igarashi K, Takizawa K, Kakushima N, et al. Should antithrombotic therapy be stopped in patients undergoing gastric endoscopic submucosal dissection? Surg Endosc 2017;31:1746-1753.

14. Furuhata T, Kaise M, Hoteya S, et al. Postoperative bleeding after gastric endoscopic submucosal dissection in patients receiving antithrombotic therapy. Gastric Cancer 2017;20:207-214.

15. Ono S, Fujishiro M, Yoshida N, et al. Thienopyridine derivatives as risk factors for bleeding following high risk endoscopic treatments: Safe Treatment on Antiplatelets (STRAP) study. Endoscopy 2015;47:632-637.

16. Ono S, Myojo M, Harada H, et al. Is it possible to perform gastric endoscopic submucosal dissection without discontinuation of a single antiplatelet of thienopyridine derivatives? Endosc Int Open 2017;5:E943-E949.

17. Kang HY, Kim SG, Kim JS, Jung HC, Song IS. Clinical outcomes of endoscopic submucosal dissection for undifferentiated early gastric cancer. Surg Endosc 2010;24:509-516.

18. Japanese Gastric Cancer Association. Japanese classification of gastric carcinoma: 3rd English edition. Gastric Cancer 2011;14:101-112.

19. Dong J, Wei K, Deng J, et al. Effects of antithrombotic therapy on bleeding after endoscopic submucosal dissection. Gastrointest Endosc 2017;86:807-816.

20. Hallas J, Dall M, Andries A, et al. Use of single and combined antithrombotic therapy and risk of serious upper gastrointestinal bleeding: population based case-control study. BMJ 2006;333:726.

21. Douketis JD, Spyropoulos AC, Spencer FA, et al. Perioperative management of antithrombotic therapy: antithrombotic therapy and prevention of thrombosis, 9th ed: American College of Chest Physicians evidence-based clinical practice guidelines. Chest 2012;141(2 Suppl):e326S-e350S.

22. Tounou S, Morita Y, Hosono T. Continuous aspirin use does not increase post-endoscopic dissection bleeding risk for gastric neoplasms in patients on antiplatelet therapy. Endosc Int Open 2015;3:E31-E38.

23. Abraham NS, Hartman C, Richardson P, Castillo D, Street RL Jr, Naik AD. Risk of lower and upper gastrointestinal bleeding, transfusions, and hospitalizations with complex antithrombotic therapy in elderly patients. Circulation 2013;128:1869-1877.

24. Holster IL, Valkhoff VE, Kuipers EJ, Tjwa ET. New oral anticoagulants increase risk for gastrointestinal bleeding: a systematic review and meta-analysis. Gastroenterology 2013;145:105-112. e15.

25. Ono S, Ono M, Nakagawa M, Shimizu Y, Kato M, Sakamoto N. Delayed bleeding and hemorrhage of mucosal defects after gastric endoscopic submucosal dissection on second-look endoscopy. Gastric Cancer 2016;19:561-567.

26. Okada K, Yamamoto Y, Kasuga A, et al. Risk factors for delayed bleeding after endoscopic submucosal dissection for gastric neoplasm. Surg Endosc 2011;25:98-107.

27. Yoshio T, Nishida T, Kawai N, et al. Gastric ESD under heparin replacement at high-risk patients of thromboembolism is technically feasible but has a high risk of delayed bleeding: Osaka University ESD study group. Gastroenterol Res Pract 2013;2013:365830.

28. Numata N, Oka S, Tanaka S, et al. Clinical outcomes of endoscopic submucosal dissection for early gastric cancer in patients with chronic kidney disease. J Gastroenterol Hepatol 2013;28:16321637.

29. Fang X, Baillargeon JG, Jupiter DC. Continued antiplatelet therapy and risk of bleeding in gastrointestinal procedures: a systematic review. J Am Coll Surg 2016;222:890-905.e11.

30. Shindo Y, Matsumoto S, Miyatani H, Yoshida Y, Mashima H. Risk factors for postoperative bleeding after gastric endoscopic submucosal dissection in patients under antithrombotics. World $\mathrm{J}$ Gastrointest Endosc 2016;8:349-356. 